\title{
Social, Academic, and Family Factors Associated With Academic Repetition in Dental Students*
}

Factores sociales, académicos y familiares asociados a la repitencia académica en estudiantes de odontología

Fatores sociais, acadêmicos e familiares associados à repetência acadêmica em estudantes de odontologia

Submission date: 25-08-2020 | Acceptance date: 28-12-2020

\section{NATALIA ForTich Mesa}

Corporación Universitaria Rafael Núñez. Cartagena, Colombia. natalia.fortich@curnvirtual.edu.co; https://orcid.org/0000-0003-2929-0278

\section{YURANIS REALES GUTIÉRREZ}

Corporación Universitaria Rafael Núñez. Cartagena, Colombia. yuranis.reales@curnvirtual.edu.co; https://orcid.org/0000-0002-2065-2810

* Original research

Correspondence: natalia.fortich@ curnvirtual.edu.co; yuranis.reales@ curnvirtual.edu.co 
doi: https://doi.org/10.11144/Javeriana.uo39.saff

How to cite: Fortich Mesa N, Reales Gutiérrez Y. Social, Academic, and Family Factors Associated With Academic Repetition in Dental Students. Univ Odontol. 2020; 39. https://doi.org/10.11144/Javeriana.uo39.saff

\section{ABSTRACT}

Background: Student repetition is a multifactorial phenomenon, of a socio-educational type, associated with different characteristics (sociodemographic, academic, family) in students of institutions, being a recurring manifestation in university institutions, which makes evident the need to identify the factors that intervene in the process, which would help to avoid or reduce dropout. Purpose: to determine the academic, social and family factors associated with student repetition in dental students from a university institution on the Colombian Caribbean coast. Methods: descriptive, cross-sectional study, in a representative sample of dental students in a repetition and non-repetition condition, who voluntarily answered a validated instrument to measure, in addition to sociodemographic characteristics, the associated factors that may influence student repetition and familiar functionality. Results: of 244 participants with a predominance of the female gender, $40,2 \%$ repeating. they never received vocational guidance $(19,4 \%)$, in general they reported good family functionality $(91 \%)$. The factors associated with student repetition were: socioeconomic status, academic levels and income of the parents, Saber11 test results and the number of times they have repeated a semester and / or a subject. Conclusion: The 
identification of these factors allows the design of strategies that encompass the impact of this condition so that higher education institutions can act in a timely manner to reduce these rates.

\section{Keywords}

academic failure; academic repetition; academic performance; academic repetition; dental; dental education; dentistry; education; higher education; students; student dropout

\section{RESUMEN}

Antecedentes: la repitencia estudiantil es un fenómeno multifactorial, de tipo socioeducativo, asociado a diferentes características (sociodemográficas, académicas, familiares) en estudiantes de instituciones, siendo una manifestación recurrente en las instituciones universitarias, que hace evidente la necesidad de identificar los factores que intervienen en el proceso, que permitiría ayudar a evitar o disminuir la deserción. Objetivo: determinar los factores académicos, sociales y familiares asociados en la repitencia estudiantil en estudiantes de odontología de una institución universitaria de la Costa Caribe colombiana. Métodos: estudio descriptivo, de corte transversal, en una muestra representativa de estudiantes de odontología en condición de repitencia y no repitencia, quienes, de forma voluntaria, respondieron instrumento validado para medir, además de las características sociodemográficas los factores asociados que pueden influir en la repitencia estudiantil y la funcionalidad familiar. Resultados: de 244 participantes con un predominio del género femenino, 40,2 \% repitentes. nunca recibieron orientación vocacional (19,4\%), en general refieren una buena funcionalidad familiar (91\%). Los factores asociados a la repitencia estudiantil 
fueron: estrato socioeconómico, niveles académicos e ingresos económicos de los padres, resultados pruebas Saber11 y el número de veces que ha repetido un semestre y/o una asignatura. Conclusión: la identificación de estos factores permite diseñar estrategias que abarquen el impacto de esta condición para que las instituciones de educación superior puedan actuar oportunamente en la reducción de estos índices.

\section{Palabras clave}

abandono escolar; educación; educación odontológica; educación superior; estudiantes de odontología; fracaso escolar; odontología; rendimiento académico; repetición académica

\section{RESUMO}

Antecedentes: A repetição de estudantes é um fenômeno multifatorial, de tipo socioeducativo ,associado a diferentes características (sociodemográficas, acadêmicas, familiares) em estudantes de instituições, sendo uma manifestação recorrente em instituições universitárias, evidenciando a necessidade de identificar os fatores que intervêm na processo, o que ajudaria a evitar ou reduzir a evasão. Objetivo: determinar os fatores acadêmicos, sociais e familiares associados à repetição de estudantes de odontologia de uma instituição universitária na costa do Caribe colombiano. Metodologia: estudo descritivo, transversal, em uma amostra representativa de estudantes de odontologia em condição de repetição e não repetição, que responderam voluntariamente a um instrumento validado para medir, além das características sociodemográficas, os fatores associados que podem influenciar a repetição do aluno e funcionalidade da família. Resultados: dos 244 
participantes com predominância do sexo feminino, 40,2 \% repetidos. nunca receberam orientação profissional $(19,4 \%)$, em geral relataram boa funcionalidade familiar $(91 \%)$. Os fatores associados à repetição do aluno foram: nível socioeconômico, nível acadêmico e renda dos pais, resultados do teste Sabre11 e o número de vezes que repetiram um semestre e / ou um assunto. Conclusão: a identificação desses fatores permite o desenho de estratégias que abranjam o impacto dessa condição, para que as instituições de ensino superior possam atuar em tempo hábil para reduzir esses índices.

\section{Palavras chave}

desempenho académico; educacão odontologica; educacão superior; estudantes de odontología; evasão escolar; fracasso académico; odontologia; repetição acadêmica

\section{INTRODUCTION}

At present, with the advent of globalization, the concept of university has been transformed until it is understood as a space in which the development of competencies (logical, interpretive, argumentative, propositional) for the adoption of roles and reflective, critical and creative attitudes that allow the resolution of problems in the social context $(1,2)$. Within this, it is important to highlight that education at the undergraduate level demands a greater demand from students to fulfill their professional training (3). However, on multiple occasions these demands are hampered by the low performance of the student, due to various factors that may be of sociodemographic, 
economic and / or related to the academic dimension, study plans and educational resources, which directly affect the repetition of the student and as a consequence of this in the student dropout (4).

For this reason, student repetition is one of the concerns shared by all educational systems in Colombia and in the world since it is the first step in the final process of university dropout. Therefore, it is considered a standard indicator below the school criteria issued by any institution, related to the minimum domain that the student must have to comply with the competencies of a subject $(5,6)$.

In the Latin American context, the situation of university education is not alien to or different from the great situation of global concern, in terms of low academic performance, university repetition and therefore student dropout (7). In the World Bank report (2017) it is estimated that countries such as Nicaragua, Panama, Bolivia, Ecuador and, unfortunately, Colombia, presented high rates of university dropouts, which suggests restructuring their educational systems in order to thus reduce rates. dropout rates and increase the quality of their educational systems (8).

For its part, in Colombia, the System for the Prevention of Drop-Out in Higher Education (SPADIES) has made progress in the centralization of information, through the development of public policies in favor of mitigating this situation, starting from the decrease in the rates of university repetition, in order to combat the high dropout rates and its negative impact on political, economic, social and cultural processes (9). Likewise, according to the Ministry of National Education (MEN), it states that the highest dropouts are found in technical and technological 
programs, and for professional programs it is $44.93 \%$. For its part, for the department of Bolívar in 2015 , the dropout rate was $44.8 \%$ (10).

Regarding the area of health sciences and specifically in the dentistry program, it has been evidenced that students refer as the main cause of repetition the short time to study, the method used by the teacher and difficulty in economic resources, which affects the quality of education due to several factors in which they can be mentioned, extra-age, that is, the gap between school age and chronological age, dropout and repetition, the latter being the highest Interest due to the fact that financial resources must be invested in students who have repeated the same subject or semester 2 or more times and therefore have not been able to be promoted, which leads to student academic failure $(11,12)$.

Other researchers such as Pérez et al, report that the causes of school dropout and repetition were absences from classes, teachers who were not very explicit in their classes, little help provided by the teacher in tutoring students, exam questions were sometimes not fit the content taught, the excessive amount of self-employment, among others (13). Also, the difficulty in financial resources by the students turns out to be the greatest inconvenience for their academic performance, followed by the absence of patients from their clinics and the lack of theoretical knowledge (14).

Based on this situation, it is necessary to make a particular identification in higher education institutions to identify the factors associated with the repetition of dental students, so that specific interventions can be carried out to reduce university dropout rates. In addition, it is essential to 
take into account that repetition generates economic effects that lead to additional costs for the family and the student, conditioning the prolongation of the professional cycle that affects the expectations of family well-being and generates possible feelings of failure, demotivation and low income. self-esteem. The need arises to determine the social, academic and family factors associated with student repetition in a dentistry program in the city of Cartagena, Colombia. For which the following research question is formulated: What are the social, academic and family factors associated with student repetition in a dentistry program in Cartagena de Indias?

\section{MATERIALS AND METHODS}

\section{Type of study}

Descriptive cross-sectional study. The population consisted of 244 students from a dental program enrolled in the first period of 2019. The following inclusion criteria were used: students over 18 years of age, in a state of repetition and non-repetition, academically active within the dentistry program and excluded students who did not accept their participation in the study through informed consent.

\section{Assessment of factors associated with repetition}

Prior to the process of carrying out the study, the students in a state of repetition and non-repetition of the dentistry program were informed of the objective and benefits of the study, based on a dialogue with the groups of students involved in order to obtain their participation. voluntarily. To 
evaluate the factors associated with repetition, the variables under study were organized according to the classification established by the (UNESCO, 2007) in; Personal (related to the emotional process and affects behavior, causing self-mortification, generating insecurity, frustrations and limitations to future life through loss of opportunities, including family members), Social and Institutional (loss of economic resources, infrastructure, human talent, logistics and finance in general) (UNESCO, 2007) (15).

A questionnaire divided into two parts was used: the first comprises an instrument validated by Muñoz and Olivera (2015) (16) which presents a Cronbach alpha of 0.709, which uses a Likerttype scale that allows measuring sociodemographic characteristics and associated factors that possibly influence the repetition process of the students, in addition the results of the ICFES Saber 11 were taken into account, in scores of 1-500 in which they were categorized into three levels: high (333-500), medium (167-332) and low (1-166). In addition, the family APGAR was applied; Suárez and Alcalá (2014) (17), a validated questionnaire for the assessment of family functionality, which presents a Cronbach alpha of 0.793 and consists of 5 questions, where each question can take a value of 0 and 4 points, which that when totaling the maximum value that can be obtained is 20 points and they were categorized into mild dysfunction, moderate dysfunction, severe diffusion and good functionality, considering that an individual has good family functionality when the score obtained is in a range of 17 to 20 points , mild dysfunction from 16 to 13 points, moderate from 12 to 9 points and severe those values less than 9 points.

\section{Statistical analysis}


For the tabulation and compilation of the information, a database was designed in the Microsoft Excel v2016 program. The information analysis was carried out using the SPSS V22 software in Spanish, using descriptive statistics by means of a univariate analysis by using the frequency and percentage measure for the qualitative variables, and measures of central tendency accompanied by dispersion measures. for continuous variables of a quantitative nature. To analyze the tendency to the statistical association between the study variables: sociodemographic and academic with the state of repetition of the population under study, a bivariate analysis was performed using Chisquare and the value of its probability was accepted as a significant statistical a value of $\mathrm{p}<0.05$.

\section{Ethical considerations}

The present study had the authorization and ethical endorsement before the ethics committee of the sponsoring institutions. Likewise, it takes into consideration regulations such as the Declaration of Helsinki and resolution 008430 of 1993 of the Ministry of Health and Social Protection of the Republic of Colombia. Informed consent was requested from the student to participate in the study, guaranteeing confidentiality and respect for dignity.

\section{RESULTS}

\section{Sociodemographic characteristics}

Of the 244 participants, $64.3 \%$ are female. Most of the participants were in an age range between 23 to 26 years, $86 \%$ of the participants were single, with a predominant socioeconomic stratum of 
stratum 2 in $39.3 \%$ and they came from the department of Bolívar and they resided in the city of Cartagena. Regarding the family environment, it was observed that $86 \%$ do not have children, $77 \%$ live with their parents and $93 \%$ do not work. The educational level of the parents is professional (32.4\%), while that of the mothers is secondary (30.7\%), apart from $50.8 \%$ of the parents have an economic income between 5 to 6 SMLV (Table 1).

TABLE 1

SOCIODEMOGRAPHIC CHARACTERISTICS OF THE POPULATION

\begin{tabular}{|c|c|c|c|c|c|c|}
\hline \multirow{2}{*}{$\begin{array}{l}\text { Características } \\
\text { sociodemográficas }\end{array}$} & \multicolumn{2}{|c|}{ Global } & \multicolumn{2}{|c|}{ Repitente } & \multicolumn{2}{|c|}{ No repitente } \\
\hline & $\mathbf{n}$ & $\%$ & $\mathbf{n}$ & $\%$ & $\mathbf{n}$ & $\%$ \\
\hline \multicolumn{7}{|l|}{ Género } \\
\hline Masculino & 87 & 35,7 & 39 & 39,8 & 48 & 32,9 \\
\hline Femenino & 157 & 64,3 & 59 & 60,2 & 98 & 67,1 \\
\hline \multicolumn{7}{|l|}{ Edad } \\
\hline 15 a 18 años & 4 & 1,6 & 0 & 0 & 4 & 2,7 \\
\hline 19 a 22 años & 81 & 33,2 & 21 & 21,4 & 60 & 41,1 \\
\hline 23 a 26 años & 109 & 44,7 & 51 & 52 & 58 & 39,7 \\
\hline 27 a 30 años & 38 & 15,6 & 20 & 20,4 & 18 & 12,3 \\
\hline Más de 30 años & 12 & 4,2 & 6 & 6,1 & 6 & 4,1 \\
\hline \multicolumn{7}{|l|}{ Estado civil } \\
\hline Soltero & 212 & 86,9 & 74 & 75,5 & 138 & 94,5 \\
\hline Casado & 17 & 7 & 11 & 11,2 & 6 & 4,1 \\
\hline Unión Libre & 15 & 6,1 & 13 & 13,3 & 2 & 1,4 \\
\hline Separado & 0 & 0 & 0 & 0 & 0 & 0 \\
\hline Viudo & 0 & 0 & 0 & 0 & 0 & 0 \\
\hline \multicolumn{7}{|c|}{ Estrato socioeconómico } \\
\hline 1 & 92 & 37,7 & 27 & 27,6 & 65 & 44,5 \\
\hline 2 & 96 & 39,3 & 46 & 46,9 & 50 & 34,2 \\
\hline 3 & 36 & 14,8 & 18 & 18,4 & 18 & 12,3 \\
\hline 4 & 4 & 1,6 & 0 & 0 & 4 & 2,7 \\
\hline 5 & 3 & 1,2 & 0 & 0 & 3 & 2,1 \\
\hline 6 & 5 & 2 & 0 & 0 & 5 & 2,4 \\
\hline 7 & 8 & 3,3 & 7 & 7,1 & 1 & 0,7 \\
\hline \multicolumn{7}{|l|}{ Número de hijos } \\
\hline 1 & 26 & 10,7 & 15 & 15,3 & 11 & 7,5 \\
\hline 2 & 5 & 2 & 3 & 3,1 & 2 & 1,4 \\
\hline 3 & 2 & 0,8 & 1 & 1 & 1 & 0,7 \\
\hline Ninguno & 211 & 86,5 & 79 & 80,6 & 132 & 90,4 \\
\hline
\end{tabular}

Convive con sus hijos 


\begin{tabular}{|c|c|c|c|c|c|c|}
\hline $\mathrm{Si}$ & 29 & 11,9 & 17 & 17,3 & 12 & 8,2 \\
\hline No & 35 & 14,3 & 34 & 34,7 & 1 & 0.7 \\
\hline No Aplica & 180 & 73,8 & 47 & 48 & 133 & 91,1 \\
\hline \multicolumn{7}{|l|}{ Convive con } \\
\hline Papá & 3 & 1,2 & 2 & 2 & 1 & 0,7 \\
\hline Mamá & 19 & 7,8 & 8 & 8,2 & 11 & 7,5 \\
\hline Ambos & 190 & 77,9 & 68 & 69,4 & 122 & 83,6 \\
\hline Pareja & 17 & 7 & 6 & 6,1 & 11 & 7,5 \\
\hline Ninguno & 15 & 6,1 & 14 & 14,3 & 1 & 0,7 \\
\hline \multicolumn{7}{|l|}{ Trabaja } \\
\hline $\mathrm{Si}$ & 16 & 6,6 & 15 & 15,3 & 1 & 0,7 \\
\hline No & 228 & 93,4 & 83 & 84,7 & 145 & 99,3 \\
\hline \multicolumn{7}{|c|}{ Lugar de procedencia } \\
\hline Antioquia & 8 & 3,2 & 1 & 1 & 7 & 4,9 \\
\hline Arauca & 1 & 0,4 & 0 & 0 & 1 & 0,7 \\
\hline Atlántico & 9 & 3,7 & 4 & 4,1 & 5 & 3,5 \\
\hline Bolívar & 142 & $\mathbf{5 7 , 5}$ & 55 & 55,8 & 87 & $\mathbf{5 9 , 7}$ \\
\hline Cesar & 9 & 3,6 & 5 & 5 & 4 & 2,7 \\
\hline Córdoba & 19 & 7,7 & 8 & 8,1 & 11 & 7,6 \\
\hline Chocó & 3 & 1,2 & 2 & 2 & 1 & 0,7 \\
\hline Cundinamarca & 1 & 0,4 & 0 & 0 & 1 & 0,7 \\
\hline La Guajira & 9 & 3,6 & 5 & 5 & 4 & 2,8 \\
\hline Magdalena & 8 & 3,2 & 4 & 4 & 4 & 2,8 \\
\hline Meta & 1 & 0,4 & 0 & 0 & 1 & 0,7 \\
\hline Norte de Santander & 1 & 0,4 & 0 & 0 & 1 & 0,7 \\
\hline Santander & 3 & 1,2 & 2 & 2 & 1 & 0,7 \\
\hline San Andrés Islas & 8 & 3,3 & 6 & 6,1 & 2 & 1,4 \\
\hline Sucre & 22 & 8,9 & 6 & 6 & 16 & 11 \\
\hline \multicolumn{7}{|l|}{ Lugar donde reside } \\
\hline Cartagena & 199 & 81,6 & 82 & 83,7 & 117 & 80,1 \\
\hline Bayunca & 10 & 4,1 & 3 & 3,1 & 7 & 4,8 \\
\hline Santa Catalina & 4 & 1,6 & 1 & 1 & 3 & 2,1 \\
\hline Clemencia & 1 & 0,4 & 0 & 0 & 1 & 0,7 \\
\hline María La Baja & 1 & 0,4 & 1 & 1 & 0 & 0 \\
\hline Otro & 29 & 11,9 & 11 & 11,2 & 18 & 12,3 \\
\hline \multicolumn{7}{|c|}{ Nivel educativo del padre } \\
\hline Ninguno & 3 & 1,2 & 3 & 3,1 & 0 & 0 \\
\hline Primaria & 20 & 8,2 & 9 & 9,2 & 11 & 7,5 \\
\hline Secundaria & 60 & 24,6 & 26 & 26,5 & 34 & 23,3 \\
\hline Técnico & 65 & 26,6 & 24 & 24,5 & 41 & 28,1 \\
\hline Tecnólogo & 17 & 7 & 12 & 12,2 & 5 & 3,4 \\
\hline Profesional & 79 & 32,4 & 24 & 24,5 & 55 & 37,7 \\
\hline Especialista & 0 & 0 & 0 & 0 & 0 & 0 \\
\hline Doctorado & 0 & 0 & 0 & 0 & 0 & 0 \\
\hline
\end{tabular}




\begin{tabular}{lcccccc}
\hline $\begin{array}{l}\text { Nivel educativo de la } \\
\text { madre }\end{array}$ & \multicolumn{7}{l}{} \\
\hline Ninguno & 1 & 0,4 & 1 & 1 & 0 & 0 \\
Primaria & 11 & 4,5 & 4 & 4,1 & 7 & 4,8 \\
Secundaria & 75 & 30,7 & 22 & 22,4 & 53 & 36,3 \\
Técnico & 70 & 28,7 & 27 & 27,6 & 43 & 29,5 \\
Tecnólogo & 19 & 7,8 & 10 & 10,2 & 9 & 6,2 \\
Profesional & 64 & 26,2 & 30 & 30,6 & 34 & 23,3 \\
Especialista & 2 & 0,8 & 2 & 2 & 0 & 0 \\
Doctorado & 2 & 0,8 & 2 & 2 & 0 & 0 \\
\hline Nivel de ingresos & & & & & & \\
económicos de los padres & 10 & 4,1 & 10 & 10,2 & 0 & 0 \\
\hline 1-2 SMLV & 22 & 9 & 22 & 22,4 & 0 & 0 \\
3-4 SMLV & 124 & 50,8 & 34 & 34,7 & 90 & 61,6 \\
5-6 SMLV & 73 & 29,9 & 26 & 26,5 & 47 & 32,2 \\
7-8 SMLV & 15 & 6,1 & 6 & 6,1 & 9 & 6,2 \\
9-10 SMLV & & & & & & \\
\hline & 244 & & 98 & & 146 & \\
\hline Total & & &
\end{tabular}

When evaluating the academic condition of the participants, it was found that $40.2 \%$ of the participants are repeaters, with an academic average of $3.49 \pm 0.37$ SD. Similarly, it was reported that they presented a medium level in the results of the Saber 11 test. However, $32.4 \%$ have repeated the semester at least once (1) and $45.5 \%$ report having repeated subjects. of the basic area (table 2).

TABLE 2

ACADEMIC FACTORS RELATED TO THE POPULATION OF STUDY

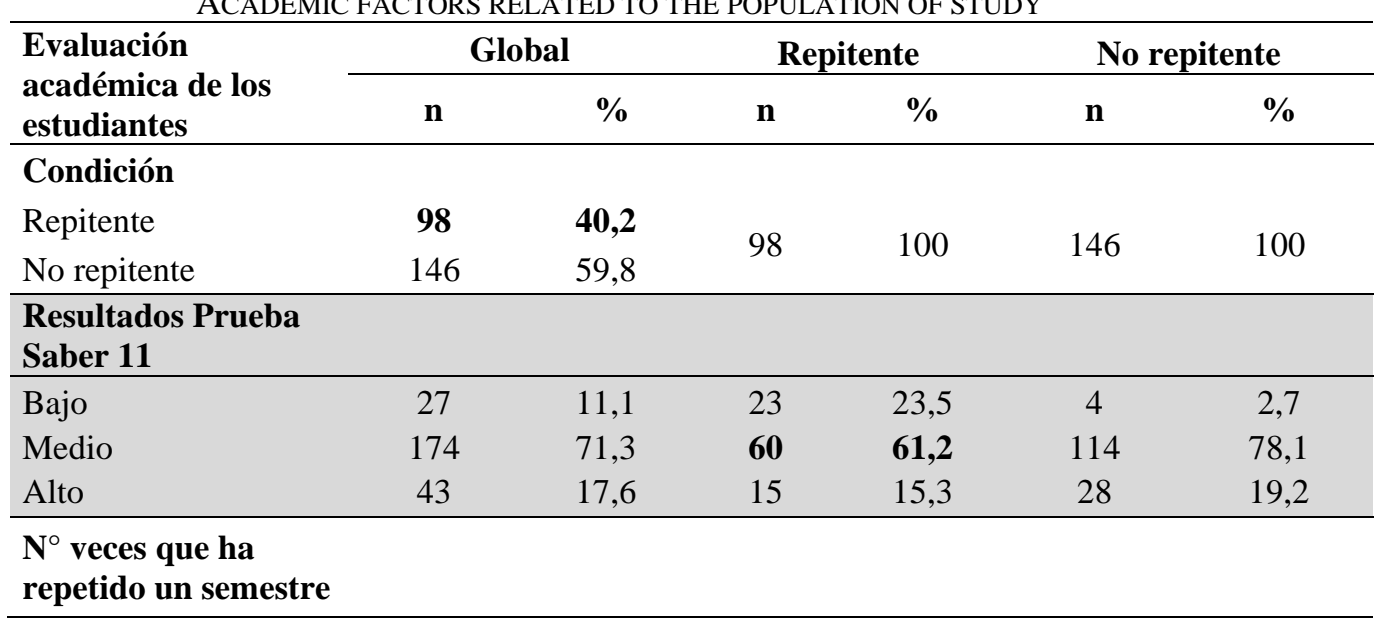




\begin{tabular}{|c|c|c|c|c|c|c|}
\hline \multirow[b]{2}{*}{0} & \\
\hline & 50 & 20,5 & 1 & 1 & 49 & 33,6 \\
\hline 1 & 79 & 32,4 & 9 & 9,2 & 70 & 47,9 \\
\hline 2 & 49 & 20,1 & 31 & 31,6 & 18 & 12,3 \\
\hline 3 & 24 & 9,8 & 20 & 20,4 & 4 & 2,7 \\
\hline 4 & 24 & 9,8 & 20 & 20,4 & 4 & 2,7 \\
\hline 5 & 6 & 2,5 & 6 & 6,1 & 0 & 0 \\
\hline 6 & 5 & 2 & 5 & 5,1 & 0 & 0 \\
\hline 7 & 2 & 0,8 & 2 & 2 & 0 & 0 \\
\hline 8 & 3 & 1,2 & 2 & 2 & 1 & 0,7 \\
\hline 9 & 1 & 0,4 & 1 & 1 & 0 & 0 \\
\hline 10 & 0 & 0 & 0 & 0 & 0 & 0 \\
\hline 11 & 1 & 0,4 & 1 & 1 & 0 & 0 \\
\hline \multicolumn{7}{|c|}{$\begin{array}{l}\mathbf{N}^{\circ} \text { veces que ha } \\
\text { repetido una } \\
\text { asignatura }\end{array}$} \\
\hline 0 & 50 & 20,5 & 0 & 0 & 50 & 34,2 \\
\hline 1 & 85 & 34,8 & 14 & 14,3 & 71 & 48,6 \\
\hline 2 & 50 & 20,5 & 30 & 30,6 & 20 & 13,7 \\
\hline 3 & 26 & 10,7 & 24 & 24,5 & 2 & 1,4 \\
\hline 4 & 18 & 7,4 & 15 & 15,3 & 3 & 2,1 \\
\hline 5 & 4 & 1,6 & 4 & 4,1 & 0 & 0 \\
\hline 6 & 4 & 1,6 & 4 & 4,1 & 0 & 0 \\
\hline 7 & 1 & 0,4 & 1 & 1 & 0 & 0 \\
\hline 8 & 4 & 1,6 & 4 & 4,1 & 0 & 0 \\
\hline 9 & 1 & 0,4 & 1 & 1 & 0 & 0 \\
\hline 10 & 0 & 0 & 0 & 0 & 0 & 0 \\
\hline 11 & 1 & 0,4 & 1 & 1 & 0 & 0 \\
\hline \multicolumn{7}{|c|}{$\begin{array}{l}\text { Asignaturas con } \\
\text { mayor repitencia }\end{array}$} \\
\hline Básicas & 111 & 45,5 & 44 & 44,9 & 67 & 45,9 \\
\hline Clínicas & 20 & 8,2 & 11 & 11,2 & 9 & 6,2 \\
\hline Ambas & 63 & 25,8 & 43 & 43,9 & 20 & 13,7 \\
\hline No Aplica & 50 & 20,5 & 0 & 0 & 50 & 34,2 \\
\hline
\end{tabular}

Regarding the factors associated with repetition, the participants answered never having received vocational guidance or having decreased their academic performance and stopped showing up due to problems related to their health or having difficulty adapting to the university environment or having family and emotional conflicts. However, between $13 \%$ to $16 \%$ rarely said they were afraid of being wrong when participating in the classroom or having a fixed schedule dedicated to their academic activities or having feelings of guilt or reviewing the topic before being seen (table 3). 
Likewise, they affirm that they have sometimes attended classes, but are tired or go to support activities at the university. Likewise, they reaffirm systematically studying the contents of the study plan, dedicating proportional time to each subject. However, between $17 \%$ to $22 \%$ of the participants commented on some occasions, feeling lack of motivation towards their career, dedicating the necessary time to their studies, presenting problems communicating, possessing previous knowledge and having difficulty memorizing the thematic contents (table 3).

Despite this, $21.4 \%$ of the students affirm that they always have support from their family group, $24.5 \%$ testify that they always feel nervousness or inner agitation at the time of evaluations, $27.6 \%$ certify that they would always obtain better grades if they proposed it and $29.6 \%$ are always convinced that studying is a very important task for their life (table 3 ).

TABLE 3

FACTORS ASSOCIATED WITH REPETITION IN THE POPULATION OF STUDY

\begin{tabular}{|c|c|c|c|c|c|c|c|c|c|c|}
\hline \multirow{2}{*}{ Global } & \multicolumn{2}{|c|}{ Nunca } & \multicolumn{2}{|c|}{$\begin{array}{l}\text { Pocas } \\
\text { veces }\end{array}$} & \multicolumn{2}{|c|}{$\begin{array}{l}\text { Algunas } \\
\text { veces }\end{array}$} & \multicolumn{2}{|c|}{$\begin{array}{c}\text { Muchas } \\
\text { veces }\end{array}$} & \multicolumn{2}{|c|}{ Siempre } \\
\hline & $\mathbf{N}$ & $\%$ & $\mathbf{n}$ & $\%$ & $\mathbf{n}$ & $\%$ & $\mathbf{n}$ & $\%$ & $\mathbf{n}$ & $\%$ \\
\hline $\begin{array}{l}\text { Recibió usted orientación vocacional e información al } \\
\text { elegir la carrera }\end{array}$ & 19 & 19,4 & 11 & 11,2 & 10 & 10,2 & 0 & 0 & 3 & 3,1 \\
\hline Ha tenido dificultad para matricularse a tiempo & 17 & 17,3 & 12 & 12,2 & 8 & 8,2 & 3 & 3,1 & 3 & 3,1 \\
\hline $\begin{array}{l}\text { Presenta dificultad para memorizar los contenidos } \\
\text { temáticos aprendidos en clases }\end{array}$ & 6 & 6,1 & 11 & 11,2 & 22 & 22,4 & 4 & 4,1 & 0 & 0 \\
\hline $\begin{array}{l}\text { Estudia sistemáticamente los contenidos que conforman } \\
\text { su plan de estudio }\end{array}$ & 6 & 6,1 & 11 & 11,2 & 15 & 15,3 & 11 & 11,2 & 0 & 0 \\
\hline $\begin{array}{l}\text { Posee conocimientos previos de los contenidos } \\
\text { temáticos desarrollados en el aula de clases }\end{array}$ & 5 & 5,1 & 7 & 7,1 & 18 & 18,4 & 10 & 10,2 & 3 & 3,1 \\
\hline Su ambiente de estudio es apropiado & 6 & 6,1 & 6 & 6,1 & 8 & 8,2 & 13 & 13,3 & 10 & 10,2 \\
\hline Dedica el tiempo necesario a sus estudios & 5 & 5,1 & 6 & 6,1 & 18 & 18,4 & 14 & 14,3 & 0 & 0 \\
\hline $\begin{array}{l}\text { Revisa la temática a tratar antes de ser vista en el aula } \\
\text { de clases }\end{array}$ & 7 & 7,1 & 16 & 16,3 & 15 & 15,3 & 4 & 4,1 & 1 & 1 \\
\hline Asiste permanentemente a sus clases & 5 & 5,1 & 2 & 2 & 3 & 3,1 & 12 & 12,2 & 21 & 21,4 \\
\hline
\end{tabular}




\begin{tabular}{|c|c|c|c|c|c|c|c|c|c|c|}
\hline $\begin{array}{l}\text { Acude a las actividades de apoyo ofrecidas por la } \\
\text { universidad para mejorar su situación académica } \\
\text { (tutorías-psicología) }\end{array}$ & 10 & 10,1 & 6 & 6,1 & 13 & 13,3 & 10 & 10,2 & 4 & 4,1 \\
\hline Siente una elevada exigencia académica en su carrera & 5 & 5,1 & 2 & 2 & 9 & 9,2 & 12 & 12,2 & 15 & 15,3 \\
\hline $\begin{array}{l}\text { Tiene un horario fijo dedicado a sus actividades } \\
\text { académicas de repaso }\end{array}$ & 6 & 6,1 & 13 & 13,3 & 12 & 12,2 & 6 & 6,1 & 6 & 6,1 \\
\hline Le resulta difícil prestar atención a algunos profesores & 12 & 12,2 & 12 & 12,2 & 12 & 12,2 & 6 & 6,1 & 1 & 1 \\
\hline Pregunta al profesor cuando no comprende algo & 6 & 6,1 & 9 & 9,2 & 8 & 8,2 & 11 & 11,2 & 9 & 9,2 \\
\hline Obtendría mejores calificaciones si se lo propusiera & 4 & 4,1 & 1 & 1 & 2 & 2 & 9 & 9,2 & 27 & 27,6 \\
\hline $\begin{array}{l}\text { El tiempo que le dedica a cada asignatura es } \\
\text { proporcional a su importancia }\end{array}$ & 5 & 5,1 & 4 & 4,1 & 15 & 15,3 & 13 & 13,3 & 6 & 6,1 \\
\hline Aprende de memoria aquellas cosas que no comprende & 6 & 6,1 & 10 & 10,2 & 12 & 12,2 & 8 & 8,2 & 7 & 7,1 \\
\hline $\begin{array}{l}\text { Te preocupas por mejorar tu calidad y velocidad en la } \\
\text { lectura }\end{array}$ & 5 & 5,1 & 3 & 3,1 & 9 & 9,2 & 9 & 9,2 & 17 & 17,3 \\
\hline $\begin{array}{l}\text { Procura relacionar, enlazar, y pensar lo que ya sabe con } \\
\text { lo que intenta aprender de memoria }\end{array}$ & 6 & 6,1 & 4 & 4,1 & 11 & 11,2 & 15 & 15,3 & 7 & 7,1 \\
\hline $\begin{array}{l}\text { Está convencido de que el estudio es una labor muy } \\
\text { importante para su vida }\end{array}$ & 5 & 5,1 & 1 & 1 & 4 & 4,1 & 4 & 4,1 & 29 & 29,6 \\
\hline $\begin{array}{l}\text { Siente nerviosismo o agitación interior en el momento } \\
\text { de las evaluaciones }\end{array}$ & 5 & 5,1 & 1 & 1 & 3 & 3,1 & 10 & 10,2 & 24 & 24,5 \\
\hline Tiene que comprobar una y otra vez todo lo que hace & 7 & 7,1 & 3 & 3,1 & 10 & 10,2 & 16 & 16,3 & 7 & 7,1 \\
\hline Le cuesta trabajo tomar decisiones & 7 & 7,1 & 10 & 10,2 & 7 & 7,1 & 15 & 15,3 & 4 & 4,1 \\
\hline Se siente decaído o falto de fuerzas & 7 & 7,1 & 4 & 4,1 & 14 & 14,3 & 15 & 15,3 & 3 & 3,1 \\
\hline Le vienen ideas de acabar con su vida & 32 & 32,6 & 4 & 4,1 & 5 & 5,1 & 1 & 1 & 1 & 1 \\
\hline Ve su futuro sin esperanzas & 23 & 23,5 & 5 & 5,1 & 11 & 11,2 & 3 & 3,1 & 1 & 1 \\
\hline Tiene sentimientos de culpa & 13 & 13,3 & 14 & 14,3 & 7 & 7,1 & 7 & 7,1 & 2 & 2 \\
\hline $\begin{array}{l}\text { Se siente incapaz de hacer las cosas o terminar las } \\
\text { tareas }\end{array}$ & 14 & 14,2 & 12 & 12,2 & 11 & 11,2 & 6 & 6,1 & 0 & 0 \\
\hline $\begin{array}{l}\text { Tiene que hacer las cosas muy despacio para estar } \\
\text { seguro de que las hace bien }\end{array}$ & 8 & 8,2 & 5 & 5,1 & 21 & 21,4 & 4 & 4,1 & 5 & 5,1 \\
\hline Se siente inferior a los demás & 27 & 27,5 & 9 & 9,2 & 5 & 5,1 & 2 & 2 & 0 & 0 \\
\hline $\begin{array}{l}\text { Tiene dificultad para adaptarse al ambiente } \\
\text { universitario }\end{array}$ & 18 & 18,4 & 10 & 10,2 & 8 & 8,2 & 5 & 5,1 & 2 & 2 \\
\hline Ha tenido intención de retirarse de la universidad & 13 & 13,3 & 6 & 6,1 & 14 & 14,3 & 9 & 9,2 & 1 & 1 \\
\hline $\begin{array}{l}\text { Se muestra inseguro y/o con miedo a no ser aceptado } \\
\text { por sus compañeros }\end{array}$ & 25 & 25,5 & 12 & 12,2 & 3 & 3,1 & 3 & 3,1 & 0 & 0 \\
\hline $\begin{array}{l}\text { Muestra poco interés y falta de motivación con las } \\
\text { asignaturas, en las que constantemente reprueba }\end{array}$ & 13 & 13,3 & 13 & 13,3 & 10 & 10,2 & 5 & 5,1 & 2 & 2 \\
\hline $\begin{array}{l}\text { Siente temor a equivocarse al participar en el aula de } \\
\text { clases cuando el docente plantea una pregunta }\end{array}$ & 8 & 8,2 & 13 & 13,3 & 7 & 7,1 & 12 & 12,2 & 3 & 3,1 \\
\hline $\begin{array}{l}\text { Se siente satisfecho con la carrera que eligió para } \\
\text { estudiar }\end{array}$ & 5 & 5,1 & 2 & 2 & 13 & 13,3 & 8 & 8,2 & 15 & 15,3 \\
\hline $\begin{array}{l}\text { Siente empatía con los docentes que le han impartido } \\
\text { clases }\end{array}$ & 4 & 4,1 & 7 & 7,1 & 13 & 13,3 & 13 & 13,3 & 6 & 6,1 \\
\hline $\begin{array}{l}\text { Tiene conflictos emocionales (soledad, disgustos } \\
\text { familiares o con su pareja) }\end{array}$ & 18 & 18,4 & 7 & 7,1 & 10 & 10,2 & 6 & 6,1 & 2 & 2 \\
\hline $\begin{array}{l}\text { Se relaciona de forma efectiva con sus compañeros y } \\
\text { docentes }\end{array}$ & 7 & 7,1 & 5 & 5,1 & 10 & 10,2 & 10 & 10,2 & 11 & 11,2 \\
\hline
\end{tabular}




\begin{tabular}{|c|c|c|c|c|c|c|c|c|c|c|}
\hline Siente falta de motivación hacia su carrera & 12 & 12,3 & 7 & 7,1 & 17 & 17,3 & 4 & 4,1 & 3 & 3,1 \\
\hline Se siente rechazado por sus compañeros & 30 & 30,6 & 7 & 7,1 & 3 & 3,1 & 3 & 3,1 & 0 & 0 \\
\hline Se siente rechazado por sus docentes & 24 & 24,5 & 8 & 8,2 & 7 & 7,1 & 4 & 4,1 & 0 & 0 \\
\hline $\begin{array}{l}\text { Presenta problemas para comunicarse y para expresar lo } \\
\text { que conoce }\end{array}$ & 8 & 8,2 & 10 & 10,2 & 20 & 20,4 & 5 & 5,1 & 0 & 0 \\
\hline Se siente identificado con su universidad & 8 & 8,2 & 8 & 8,2 & 10 & 10,2 & 13 & 13,3 & 4 & 4,1 \\
\hline $\begin{array}{l}\text { En su ambiente familiar son frecuentes los conflictos } \\
\text { familiares (disgustos con sus padres y hermanos) }\end{array}$ & 17 & 17,4 & 11 & 11,2 & 4 & 4,1 & 9 & 9,3 & 2 & 2 \\
\hline Tiene apoyo por parte de su grupo familiar & 5 & 5,1 & 0 & 0 & 3 & 3,1 & 14 & 14,3 & 21 & 21,4 \\
\hline $\begin{array}{l}\text { Se ha ausentado de clases frecuentemente por causa de } \\
\text { problemas relacionados con su salud }\end{array}$ & 16 & 16,4 & 9 & 9,2 & 5 & 5,1 & 10 & 10,2 & 3 & 3,1 \\
\hline $\begin{array}{l}\text { Ha dejado de presentar evaluaciones y trabajos } \\
\text { importantes a causa de una enfermedad }\end{array}$ & 16 & 16,4 & 12 & 12,2 & 9 & 9,2 & 3 & 3,1 & 3 & 3,1 \\
\hline $\begin{array}{l}\text { Asiste a clases, pero se muestra cansado y fatigado } \\
\text { durante el desarrollo de éstas }\end{array}$ & 8 & 8.2 & 11 & 11.2 & 13 & 13,3 & 11 & 11,2 & 0 & 0 \\
\hline $\begin{array}{l}\text { Ha disminuido su rendimiento académico debido a } \\
\text { problemas de salud }\end{array}$ & 15 & 15,3 & 15 & 15,3 & 5 & 5.1 & 6 & 6.1 & 2 & 2 \\
\hline
\end{tabular}

In relation to family functionality (family APGAR) in the study population, it could be determined that the participants presented good family functionality in $91 \%$, while only $9 \%$ presented mild dysfunction (table 4).

TABLE 4

FAMILY FUNCTIONING IN THE POPULATION OF STUDY

\begin{tabular}{|c|c|c|c|c|c|c|c|c|c|c|}
\hline \multirow[t]{2}{*}{ APGAR familiar } & \multicolumn{2}{|c|}{ Nunca } & \multicolumn{2}{|c|}{$\begin{array}{c}\text { Casi } \\
\text { nunca }\end{array}$} & \multicolumn{2}{|c|}{$\begin{array}{l}\text { Algunas } \\
\text { veces }\end{array}$} & \multicolumn{2}{|c|}{$\begin{array}{l}\text { Muchas } \\
\text { veces }\end{array}$} & \multicolumn{2}{|c|}{ Siempre } \\
\hline & $\mathbf{n}$ & $\%$ & $\mathbf{n}$ & $\%$ & $\mathbf{n}$ & $\%$ & $\mathbf{n}$ & $\%$ & $\mathbf{n}$ & $\%$ \\
\hline $\begin{array}{l}\text { Me satisface la ayuda que recibo de mi familia } \\
\text { cuando tengo algún problema o necesidad }\end{array}$ & 0 & 0 & 1 & 2,3 & 7 & 16,3 & 10 & 23,3 & 25 & 58 \\
\hline $\begin{array}{l}\text { Me satisface la participación que mi familia me } \\
\text { brinda y me permite }\end{array}$ & 0 & 0 & 1 & 2,3 & 3 & 7,0 & 14 & 32,6 & 25 & 58 \\
\hline $\begin{array}{l}\text { Me satisface cómo mi familia acepta y apoya mis } \\
\text { deseos de emprender nuevas actividades }\end{array}$ & 0 & 0 & 1 & 2,3 & 5 & 11,6 & 13 & 30,2 & 24 & 56 \\
\hline $\begin{array}{l}\text { Me satisface cómo mi familia expresa afectos y } \\
\text { responde a mis emociones como rabia, tristeza, } \\
\text { amor, etc. }\end{array}$ & 1 & 2,3 & 0 & 0 & 12 & 27,9 & 16 & 37,2 & 14 & 33 \\
\hline $\begin{array}{l}\text { Me satisface cómo compartimos en mi familia: a) el } \\
\text { tiempo para estar juntos b) los espacios de la casa c) } \\
\text { el dinero }\end{array}$ & 1 & 2,3 & 3 & 7 & 12 & 27,9 & 14 & 32,6 & 13 & 30 \\
\hline
\end{tabular}

\begin{tabular}{lcc}
\hline Funcionalidad & n & \% \\
\hline Buena funcionalidad & 39 & 91 \\
Disfunción leve & 4 & 9
\end{tabular}




\begin{tabular}{lll} 
Disfunción moderada & 0 & 0 \\
Disfunción severa & 0 & 0 \\
\hline
\end{tabular}

Finally, when correlating the sociodemographic and academic variables with the academic condition of the students through the bivariate analysis with Pearson's Chi-square test, it was possible to conclude that most of the socio-demographic variables evaluated significantly influence the academic condition of the students. the students (table 5).

TABLE 5

ASSOCIATION BETWEEN SOCIODEMOGRAPHIC FACTORS AND ACADEMIC CONDITION IN THE POPULATION OF STUDY

\begin{tabular}{|c|c|c|c|}
\hline \multirow{2}{*}{$\begin{array}{l}\begin{array}{l}\text { Características } \\
\text { sociodemográficas }\end{array} \\
\text { Género }\end{array}$} & \multicolumn{2}{|c|}{ Condición académica } & \multirow[t]{2}{*}{ Valor de $\mathrm{p}$} \\
\hline & Repitente & No repitente & \\
\hline Masculino & 39 & 48 & \multirow{2}{*}{0,269} \\
\hline Femenino & 59 & 98 & \\
\hline \multicolumn{4}{|l|}{ Estado civil } \\
\hline Soltero & 74 & 138 & \multirow{3}{*}{$0,000 *$} \\
\hline Casado & 11 & 6 & \\
\hline Unión Libre & 13 & 2 & \\
\hline \multicolumn{4}{|c|}{ Estrato socioeconómico } \\
\hline 1 & 27 & 65 & \multirow{7}{*}{$0,001 *$} \\
\hline 2 & 46 & 50 & \\
\hline 3 & 18 & 18 & \\
\hline 4 & 0 & 4 & \\
\hline 5 & 0 & 3 & \\
\hline 6 & 0 & 5 & \\
\hline 7 & 7 & 1 & \\
\hline \multicolumn{4}{|c|}{ Convive con sus hijos } \\
\hline $\mathrm{Si}$ & 17 & 12 & \multirow{2}{*}{$0,000 *$} \\
\hline No & 34 & 1 & \\
\hline \multicolumn{4}{|l|}{ Convive con } \\
\hline Papá & 2 & 1 & \multirow{5}{*}{$0,001^{*}$} \\
\hline Mamá & 8 & 11 & \\
\hline Ambos & 68 & 122 & \\
\hline Pareja & 6 & 11 & \\
\hline Ninguno & 14 & 1 & \\
\hline \multicolumn{4}{|l|}{ Trabaja } \\
\hline $\mathrm{Si}$ & 15 & 1 & \multirow{2}{*}{$0,000 *$} \\
\hline No & 83 & 145 & \\
\hline
\end{tabular}




\begin{tabular}{lccc}
\hline $\begin{array}{l}\text { Nivel educativo del } \\
\text { padre }\end{array}$ & & & \\
\hline Ninguno & 3 & 0 & $011^{*}$ \\
Primaria & 9 & 11 & \\
Secundaria & 26 & 34 & \\
Técnico & 24 & 41 & \\
Tecnólogo & 12 & 5 & $0,057^{*}$ \\
Profesional & 24 & 46 & \\
\hline Nivel educativo de la & & & \\
madre & & 0 & \\
\hline Ninguno & 1 & 7 & \\
Primaria & 4 & 53 & \\
Secundaria & 22 & 43 & \\
Técnico & 27 & 9 & \\
Tecnólogo & 10 & 34 \\
Profesional & 30 & 0 & \\
Especialista & 2 & 0 & \\
Doctorado & 2 & & \\
\hline Nivel de ingresos & & 0 \\
económicos de los padres & & 0 & \\
\hline 1-2 SMLV & 10 & 90 \\
3-4 SMLV & 22 & 47 \\
5-6 SMLV & 34 & 9 & \\
7-8 SMLV & 26 & 6 & \\
9-10 SMLV & & & \\
\hline
\end{tabular}

Regarding academic factors, statistically significant associations were reported between the level of Saber 11 results and the number of times the student has repeated a semester and / or a subject $(p=0.000)$ respectively (table 6$)$.

TABLE 6

ASSOCIATION BETWEEN ACADEMIC FACTORS AND ACADEMIC CONDITION IN THE POPULATION OF STUDY

\begin{tabular}{llll}
\multirow{2}{*}{$\begin{array}{l}\text { Evaluación académica. } \\
\text { Resultados Saber 11 }\end{array}$} & \multicolumn{2}{c}{ Condición académica } & \multirow{2}{*}{ Valor de p } \\
\cline { 2 - 3 } & Repitente & No repitente & \\
\hline Bajo & 23 & 28 & $0,000^{*}$ \\
Medio & 60 & 114 & \\
Alto & 15 & 4 & \\
\hline $\mathbf{N}^{\circ}$ de veces que ha & & & \\
repetido un semestre & & & $0,000^{*}$ \\
\hline 0 & 1 & 49 &
\end{tabular}




\begin{tabular}{llll}
1 & 9 & 70 \\
2 & 31 & 18 \\
3 & 20 & 4 & \\
4 & 20 & 4 \\
5 & 6 & 0 \\
6 & 5 & 0 & \\
7 & 2 & 0 & \\
8 & 2 & 1 & \\
9 & 1 & 0 & \\
10 & 0 & 0 & \\
11 & 1 & 0 & \\
\hline Evaluación académica & Condición académica de p \\
& & & \\
\hline $\mathbf{N}^{\circ}$ de veces que ha & Repitente & No repitente & \\
repetido una asignatura & & & \\
\hline 0 & 0 & 50 & \\
1 & 14 & 71 & \\
2 & 30 & 20 & \\
3 & 24 & 2 & \\
4 & 15 & 3 & \\
5 & 4 & 0 & \\
6 & 4 & 0 & \\
7 & 1 & 0 & \\
8 & 4 & 0 & \\
9 & 1 & 0 & \\
10 & 0 & 0 & \\
11 & 1 & 0 & \\
\hline
\end{tabular}

\section{DISCUSSION}

The present study allowed the sociodemographic characterization of the study subjects and their relationship with repetition in dental students of a particular program, however, despite the fact that these results cannot be generalized to all dental schools, if they agree to identify Factors that may be common to all dentistry programs, which would allow the design of improvement actions in favor of quality indicators and the reduction of desertion in university students in Health Sciences Programs. In the present work, repetition was evaluated, as it was understood as the action of repeatedly pursuing an academic activity, either due to poor student performance or due to 
causes beyond the academic field during a period (year, semester or quarter $(18,19)$. It is necessary to identify the academic, social and family factors associated with student repetition in a dental program in Cartagena de Indias, an interest similar to research by authors such as Dadpe et al and Graffigna et al $(20,21)$.

Yepes-Delgado et al identified the main causes of academic dropout in $37.5 \%$ of cases to academic difficulties, $31.3 \%$ occurred due to program change, $18.8 \%$ due to economic reasons or work reasons. On the other hand, re-entry students lost the courses due to academic difficulties in $38.9 \%$, $27.8 \%$ due to work reasons and $33.3 \%$ due to physical or mental health reasons - lack of selfesteem (22). Results that differ from those present, where the students stated that they never stopped attending the university for health reasons. However, they maintain that on some occasions they are tired and attend university support programs because they intend to withdraw from the academic program. Similar data are reported by Medina and Gaytán, who identified the factors that have influenced the students of the Faculty of Dentistry of the UACH to abandon their professional studies, of which the physical and emotional discomfort due to the excessive burden of work on theoretical subjects, clinical practices that significantly affect the health and well-being of students (23).

Flores M, et al state that the environments that most generate anxiety responses in dental students are associated with exam and public speaking situations, which is why they suggest that the forms of evaluation should be modified, promoting better communication between students. teachers and students, if possible, sensitize teachers to try to be more understanding and caring with their students (24). Similar results to the present investigation, $24.5 \%$ of the participants stated that they 
were always nervous or agitated at the time of the evaluations and $20.4 \%$ had problems communicating and / or expressing themselves and only $13.3 \%$ of the students reported feeling empathy for the teachers who taught them.

Rueda S, et al determined the dropout risk profile according to socioeconomic, institutional, academic, and individual conditions in students from the headquarters of a Colombian university, reporting that $87.5 \%$ of the participants were single, $81.1 \%$ belonged to socioeconomic strata 1 2 and the academic average was 3.7, according to institutional conditions, $0.8 \%$ of the students have a desire to withdraw from the institution. However, the participants report having good relationships with friends in the university environment and seeking support when required. With regard to family aspects, $16.2 \%$ affirmed having children, $33.3 \%$ were related to living in nuclear families, and $12.8 \%$ perceived having severe family dysfunction (25), a relationship can be evidenced with results regarding the academic average (3.49), marital status (single), socioeconomic stratum (stratum 1 and 2), in relation to family functionality it was found that $91 \%$ of students have good family functionality, not However, $14.3 \%$ of the participants in the present study stated that they intended to withdraw from the university.

Finally, Díaz S, et al associated academic stress and family functionality in dental students, reporting that among the situations that generate stress are teacher evaluations, restlessness, despair, drowsiness and lack of organization of academic activities. Regarding family functionality, the APGAR reported good family functionality in $38.25 \%$, followed by mild dysfunction in $31.8 \%$ (26). When relating these findings to the present study, a relationship was found because $13.3 \%$ attend classes, but are tired and fatigued about its development and $18.4 \%$ 
only sometimes dedicate the necessary time to their studies or academic activities. On the contrary, this study reported that the participants have good family functionality, which is considered an important factor on school repetition due to aspects such as type of family, educational level, economic income and the support of the family towards their children. / as can affect the academic condition of students.

However, an important limitation corresponds to the design of the cross-sectional study, for future investigations the implementation of longitudinal studies is suggested, which allows obtaining complementary information and thus carrying out the necessary interventions since student repetition is linked to emotional factors, which are fluctuating and changing over time.

The results obtained in this research were presented to the academic authorities of the Program for their evaluation and the taking of improvement actions.

\section{CONCLUSION}

The identification of factors associated with repetition in students will allow establishing new teaching and learning methodologies to increase effectiveness and motivation during their academic training, as well as higher education institutions so that they can act in a timely manner in the reduction of these indices. Therefore, it is necessary to follow up students who drop out or show recurrent repetition during the training of the degree.

\section{ACKNOWLEDGMENT}


The authors thank the School of Education of the Technological University of Bolívar, led by Dr. William Arellano Cartagena for the support in this research work and the study participants.

\section{REFERENCES}

1. García-Jiménez E. Concepto de excelencia en enseñanza superior universitaria. Educación médica. 2016 jul-sep; 17(3): 83-87. https://doi.org/10.1016/j.edumed.2016.06.003

2. Melo-Becerra LA, Ramos-Forero JE, Hernández-Santamaría PO. La educación superior en Colombia: situación actual y análisis de eficiencia. Revista Desarrollo y sociedad. 2017 enejun; (78): 59-111. https://doi.org/10.13043/dys.78.2

3. Pérez MV, Valenzuela - Castellanos M, Díaz A, González-Pienda, JA, Núñez JC. Dificultades de aprendizaje en estudiantes universitarios de primer año. Atenea. 2013; 508( II Sem): 135150. https://dx.doi.org/10.4067/S0718-04622013000200010

4. Velázquez - Narváez Y, González - Medina M. Factores asociados a la permanencia de estudiantes universitarios: caso UAMM-UAT. Revista de la Educación Superior. 2017 oct; 46(184): 117-138. https://doi.org/10.1016/j.resu.2017.11.003

5. Gartner, ML, Dussán C, Montoya DM. Caracterización de la deserción estudiantil en la Universidad de Caldas el período 2009-2013. análisis a partir del Sistema para la Prevención de la Deserción de la Educación Superior-SPADIES. Revista Latinoamericana de Estudios Educativos. 2016 ene- jun; 12(1): 132-158.

6. Romero- Navarro L, García - Pérez E, Ramos - Rodríguez C, Martínez - Enamorado M. La repitencia: un reto para la Educación Médica guantanamera. Revista de Información Científica 
2017; 96(6): 1036-1045.

7. Rodríguez, M. La investigación sobre deserción universitaria en Colombia 2006-2016. Tendencias y resultados. Pedagogía y Saberes. 2016 jun; 51: 49-66. https://doi.org/10.17227/pys.num51-8664

8. Ferreyra M, Avitabile C, Álvarez J, Haimovich, F, Urzúa, S. Momento decisivo: la educación superior en América Latina y el Caribe. Washington, DC: Banco Mundial; 2017.

9. Munizaga - Mellado F, Cifuentes - Orellana M, Beltrán - Gabrie, A. Variables y factores asociados al fenómeno de la retención y abandono estudiantil universitario en investigaciones de Latinoamérica y el Caribe. Congresos CLABES VII, Córdoba - Argentina 2017.

10. República de Colombia. Ministerio de Educación Nacional - SPADIES [Internet]. Estadísticas - Deserción y Graduación. 2015

11. Aragon BL, Chávez BM, Flores TMA. Evaluación de la ansiedad en estudiantes de la carrera de cirujano dentista. Revista Digital Internacional de Psicología y Ciencia Social. 2015 feb; 1(1): $33-42$.

12. Quintero V, Zárate N. Autoestima, autoconcepto y su relación con el rendimiento académico en estudiantes de odontología. Revista de Sistemas y Gestión Educativa. 2016 dic; 3(9): 1119.

13. Pérez - Cardoso C, Cerón - Mendoza E, Suárez - Mella R, Mera - Martínez M, Briones Bermeo N, Zambrano - Loor L, et al. Deserción y repitencia en estudiantes de la carrera de Enfermería matriculados en el período 2010-2015. Universidad Técnica de Manabí. Ecuador. 2017. Educacion $\quad$ Médica. 2019 Mar- Abr; 20(2), 84-90. https://doi.org/10.1016/j.edumed.2017.12.013

14. García L, Moreno V. Factores asociados al rendimiento académico de los estudiantes de 
odontología en la clínica de crecimiento y desarrollo. Acta Odontol. Colomb [Internet]. 2017 Ene [citado 27 de junio]; 7(1): 81-89.

15. UNESCO. Estudio sobre repitencia y deserción universitaria en América Latina 2000-2005.

16. Muñoz R, Olivera M. Repitencia del programa de medicina en una institución de Educación Superior en la ciudad de Cartagena (Tesis de maestría) Cartagena, Colombia Universidad Tecnológica de Bolívar; 2015

17. Suárez MA, Alcalá M. Apgar Familiar: Una Herramienta Para Detectar Disfunción Familiar. Rev Méd La Paz [Internet] 2014 ene- jun; 20(1): 53-57.

18. Torres-González, J, Acevedo-Correa D, Gallo-García L. Causas y consecuencias de la deserción y repitencia escolar: una visión general en el contexto latinoamericano. Cul Edu Soc. 2015; 6(2): 157-187.

19. Jiménez-Herrera, L. Reflexiones teóricas del fenómeno de repitencia, prolongación, abandono y deserción de estudiantes universitarios. Revista Pensamiento Actual. 2016; 16(27): 261-269.

20. Dadpe AM, Shah DY, Vinay V, Shetkar P. Factors Facilitating Academic Success in Dental Students After Initial Failure: A Qualitative Study. J Dent Educ. 2018; 82(11): 1155-1161. https://doi.org/10.21815/JDE.018.119

21. Graffigna AM, Morell M, Simonassi ML, Morales A. Determinants to retain students during the first year of university studies. Procedia - Social and Behavioral Sciences. 2014; 116 : 2630-2634. https://doi.org/10.1016/j.sbspro.2014.01.625

22. Yepes-Delgado F, Beltrán-Salazar M, Martínez-Ceferino E. Academic dropout among undergraduate students from Universidad de Antioquia School of Dentistry, 2004-2014. Rev Fac Odontol Univ Antioq. 2017 Jul- Dic; 29(1): 7695. https://doi.org/10.17533/udea.rfo.v29n1a5 
23. Medina - Soto MD, Gaytán - Díaz CC. Factores y significados de la deserción escolar en el nivel superior. Caso: Facultad de Odontología de la UACH. RECIE. Revista Electrónica Científica de Investigación Educativa. 2018 Sep; 4(1): 515-525.

24. Flores-Tapia MA, Chávez-Becerra M, Aragón Borja, LE. Situaciones que generan ansiedad en estudiantes de Odontología. Journal of Behavior, Health \& Social Issues. 2016 Nov; 8(2): 3541. http://dx.doi.org/10.1016/j.jbhsi.2016.11.004

25. Rueda-Ramírez SM, Urrego-Velásquez D, Páez-Zapata E, Velásquez-C, Hernández-Ramírez, EM. Perfiles de riesgo de deserción en estudiantes de las sedes de una universidad colombiana. Revista de Psicología. 2020; 38(1): 275-297. https://doi.org/10.18800/psico.202001.011

26. Díaz- Cárdenas S, Martínez-Redondo M, Zapata-Teherán AM. Rendimiento académico y calidad de vida relacionada con la salud en estudiantes de odontología. SaludUninorte 2017; 33(2): 139-151 